\title{
WOOD ANATOMICAL CLASSIFICATION USING ITERATIVE CHARACTER WEIGHING*
}

\author{
P. HOGEWEG AND J. KOEK-NOORMAN
}

Afdeling voor Theoretische Biologie and Instituut voor Systematische Plantkunde, Utrecht

\section{SUMMARY}

In this paper we investigate the pattern of wood anatomical variation in some groups of Rubiaceae (i.e. Cinchoneae, Rondeletieae and Condamineae) by using a numerical pattern detection method which involves character weighing (HoGEwEG 1975). In this method character weights are obtained iteratively on the basis of the distribution of character values in previously generated classes; these classes being generated by agglomerative cluster analysis, initially with characters weighed equally and subsequently with characters weighed differentially as indicated above. The result of such a study consists of a sequence of dendrograms together with the character weights by which these are produced. Our biological conclusions include:

The so obtained results confirm the conclusions drawn by KoEK-NOORMAN \& Hogeweg (1974) that the pattern of variation in the wood anatomical structure of these taxa is consistent with the existing classifications at the genus level but does not warrant the higher level classification in Cinchoneae, Rondeletieae and Condamineae as each of these groups show two majorly different woodtypes (differing most conspicuously with respect to libriform fibres and fibre tracheids) while these wood types constitute the major pattern of variation in the sample.

However, during the iteration, the Rondeletieae and Cinchoneae tend to be distinguished as separate groups on a level below this main bipartition. The Condamineae remain scattered.

The genus Cinchona becomes sharply separated from the other groups during the iteration. Its intermediate position with respect to fibre tracheids and libriform fibres was noted prior to this analysis by Koek-Noorman (KOEK-NOORMAN \& HOGEWEO 1974).

Rather surprisingly, ambiguities (in the sense of being differently classified by different authors) in the existing classifications based mainly on flower morphology reoccur in our iteration based on wood anatomical data in the sense that these taxa change position in the later steps of the iteration.

Finally we note that character weights obtained by our method do not spoil the grouping in genera as did character weights obtained by considering the literature on evolutionary trends in wood anatomy (KOEK-NOORMAN \& HOGEWEG 1974), and that the character weights obtained by our method are not counter to intuition.

\section{INTRODUCTION}

In this paper we elaborate on a numerical taxonomic study of the wood anatomical variation in some groups of Rubiaceae, i.e. Cinchoneae, Rondeletieae and Condamineae, which was published previously (KOEK-NOORMAN \& HogEWEG 1974). It was shown at that time that the close resemblance which is generally found between the pattern of variation as expressed in the existing classifications (based mainly on flower characteristics) and the variation in the wood

* Mededelingen van het Botanisch Museum en Herbarium van de Rijksuniversiteit te Utrecht No. 424. 
anatomy, breaks down, at the level of tribes, in these groups. The main pattern of variation in the wood anatomy was with respect to fibre characters, resulting in two major wood types. Both these wood types occur in all the three groups mentioned above. The genera were, however, clustered quite well indicating a similar pattern of variation in wood and flower characters at the genus level. We also reported in the above mentioned paper that working the other way around, i.e. starting with the above mentioned tribes we could find only a very few characters which were unevenly distributed over the groups; these characters were moreover intuitively not very satisfactory as they show a large within specimen variation. Especially in this latter i.e. "supervised" approach we should, however, keep in mind that the classifications published until now are controversial in some points (see e.g. BREMEKAMP (1966), KoEK-NoORMAN (1969), Verdcourt (1958), STEYermark (1972) ${ }^{1}$ ). The present study employs therefore entirely "non-supervised" methods.

All details about the material used, as well as a complete specification of the entire character set used in this study, may be found in KOEK-NOORMAN \& HoGEWEG (1974). Here we will continue this analysis by using a numerical method proposed by HoGEWEG (1975), which generates a weighing of the characters during its execution.

\section{METHOD}

The method was described in full in HoGEwEG (1975).

We will here confine ourselves to present the general motivation which led to the formulation of the method together with an outline of the method.

The method was conceived with the following points in mind:

1. Cluster analysis (as well as other pattern detection methods) is not feasible without some kind of weighing of characters, as has been proved by WATANABE (1969). The opposite has often been claimed in the context of numerical taxonomy. It may even be said that the use of numerical pattern detection techniques in taxonomy has risen from the desire to ban character weighing from taxonomy (Sokal \& SNeath 1963, SNEATH \& Sokal 1973). In practice, therefore, the character weighing in numerical taxonomic studies has been kept largely implicit and is confined to a "zero-one" weighing, i.e. to whether a character is included in the analysis or not. However, as character weighing is unavoidable we might as well incorporate it in an explicitised procedure.

2. Closely connected with point 1 we note that the form of the results in a numerical taxonomic study and a classical taxonomic study are quite different. The former emphasizes the procedure which led to a grouping of the objects while the latter is mainly concerned with the evaluation of characters with

\footnotetext{
${ }^{1}$ For instance, the tribes Condamineae and Rondeletieae as circumscribed by SchumanN (1897) are combined to a single tribe Rondeletieae by Verdcourt. Bremekamp splits the Rondeletieae sensu stricto in Rondeletieae, Simireae (with the sole genus Simira) and Gleasonieae (to accomodate the genus Gleasonia).
} 
respect to their power in distinguishing between, implicitly defined, groups. The latter point is stated explicitly by LEENHOUTS (1968):

"the material is sorted into groups prior to careful analysis and description." The grouping may be adjusted later on in accordance with the description. Looked upon this way, the analysis as done in numerical taxanomy and in classical taxonomy constitute two different steps in the entire taxonomic analysis.

The method which will be used in this paper combines both these steps in one, numerical, procedure.

Starting with a fixed 'scope of observation' (character choice remains implicit here) we weigh the characters equally for lack of further knowledge. We obtain a grouping of objects by agglomerative cluster analysis followed by optimal partitioning of the dendrogram and evaluate all characters in the scope of observation as to the extent to which they support the proposed grouping, i.e. to the extent to which they are unequally distributed among the groups. Next the characters are weighed accordingly and a new grouping is obtained, again by agglomerative cluster analysis. This is repeated several times, the results consisting of a sequence of dendrograms together with the character weights which generated them. In fig. $I$ the method is shown schematically. It is implemented in BIOPAT, Program system for Biological Pattern Analysis (HoGEWEG \& HeSPER 1972). Properties of the method were shown to be (HogeweG 1975): 1. The pattern became more pronounced.

2. The resulting classification converged to previously proposed classifications.

3. The method sorted out conflicting patterns of variation in the dataset.

Here we will apply the method on the wood anatomical dataset mentioned above and compare the results with our own judgement on the affiliation of the wood anatomical structures and with known classifications based on flower morphology.

\section{RESULTS}

\subsection{Protocol of Iterative Weighing Procedure}

The results are given in the form of a protocol below and in fig. 2 and 3 showing respectively the sequence of dendrograms and a profile of character weights.

Step 1

Weight $=1$

for all characters.

Dendrogram: see fig. $2 a$.

This dendrogram is different from the one published in KOEK-NoORMAN \& HOGEWEG (1974) because then invariant characters were left out. The exclusion of invariant characters yields a different result because the characterset contains not-observed characters, which gives rise to locally diminished dimensionality in the analysis and therefore renders it dependent on total number of dimensions. 


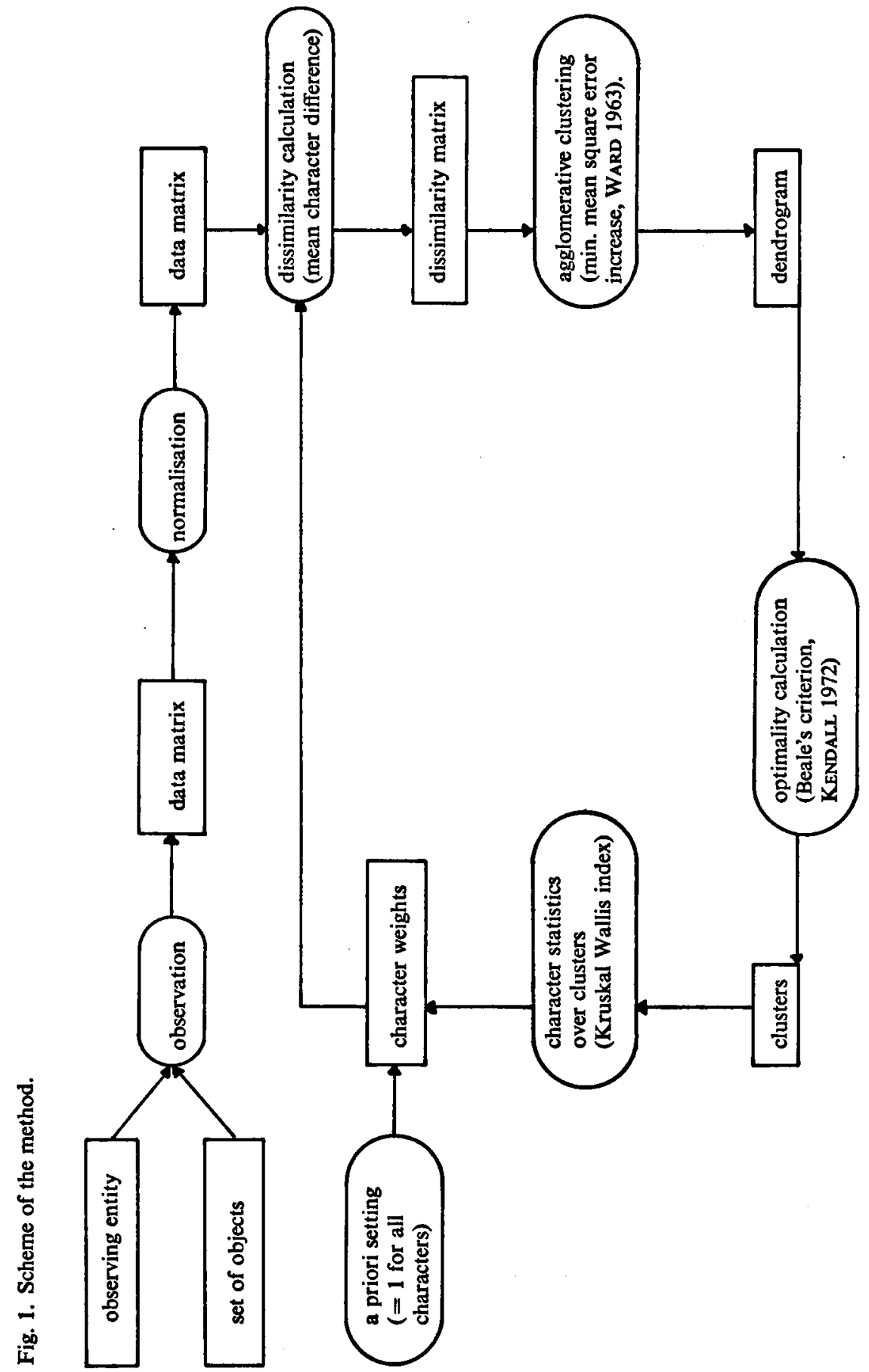


Clusters:

Optimal partitioning (Beale's criterion, see HogEwEG 1975) of 1 cluster yields 3 clusters (value $=112$ )

Step 2

Weights ( fig. 3):

High weights in several characters: arrangement of the vessels, fibre characters, and the presence of apotracheal parenchyma.

Dendrogram: see fig. $2 b$.

The structure is the same on the level of bipartition. The most striking change, as compared to step 1, is the emergence of the genus Cinchona as a sharply distinguished cluster. The aberrant wood anatomy of this genus had been noticed by Koek-Noorman prior to the analysis.

\section{Clusters:}

Optimal partitioning of 3 clusters yields 6 clusters (value $=17$ ). Good partitionings of 1 cluster is in 2, 3, 4, 5, 6, 9 clusters with optimality value of 409, 407,367, 345,345 respectively. (Note the high increase of these values as compared to step 1).

\section{Step 3 \\ Weights (fig. 3):}

Large increase of weights as compared to step 2 are mainly in shape, diameter and number of vessels, cell wall thickness, ray-vessel pitting, and the presence of scanty paratracheal parenchyma.

Dendrogram: see fig. $2 c$.

The 4 largest groups remain equal. A subgroup of Cinchoneae (Ferdinandusa, Dolicholobium, Capirona, Macbrideina (together with Chimarrhis (Condamineae)) emerges with very distinct identity.

Clusters:

Optimal partitioning of 6 clusters yields 8 clusters (value $=11$ ). One cluster may be partitioned in $2,4,6,5,8$ clusters with respective optimality values 340 , $322,300,298,296$.

Step 4

Weights (fig. 3):

Largest increase in weight as compared to step 3 occurs in the size of the vessel and wall perforations, number and diameter of the vessels, length of vessel members, width and height of rays, sheath cells, the presence of square/upright cells and of crystals, and of paratracheal parenchyma.

Dendrogram: see fig. $2 d$.

In this step the Rondeletieae s.l. tend to establish themselves as a separate group in both clusters of the above mentioned bipartition (only 2 exceptions in each of them). This was true for one half of the bipartition all through the iteration.

Clusters:

Optimal partitions of 8 clusters yield 9 clusters at value $=7$. One cluster may be subdivided in $2,5,8,6,9$ clusters with optimality values of $240,223,221,220$, 216 respectively (note the marked decrease as compared to step 2 and 3). However, the iteration was pursued one more step. 


\title{
DENDROGRAM (WARD RVERAGES) OF MEAN CHARACTER DIFFERENCES IN Q-MODE
}

\author{
RUBIRCEAE, WOODANATUMY \\ $C Y=0$
}

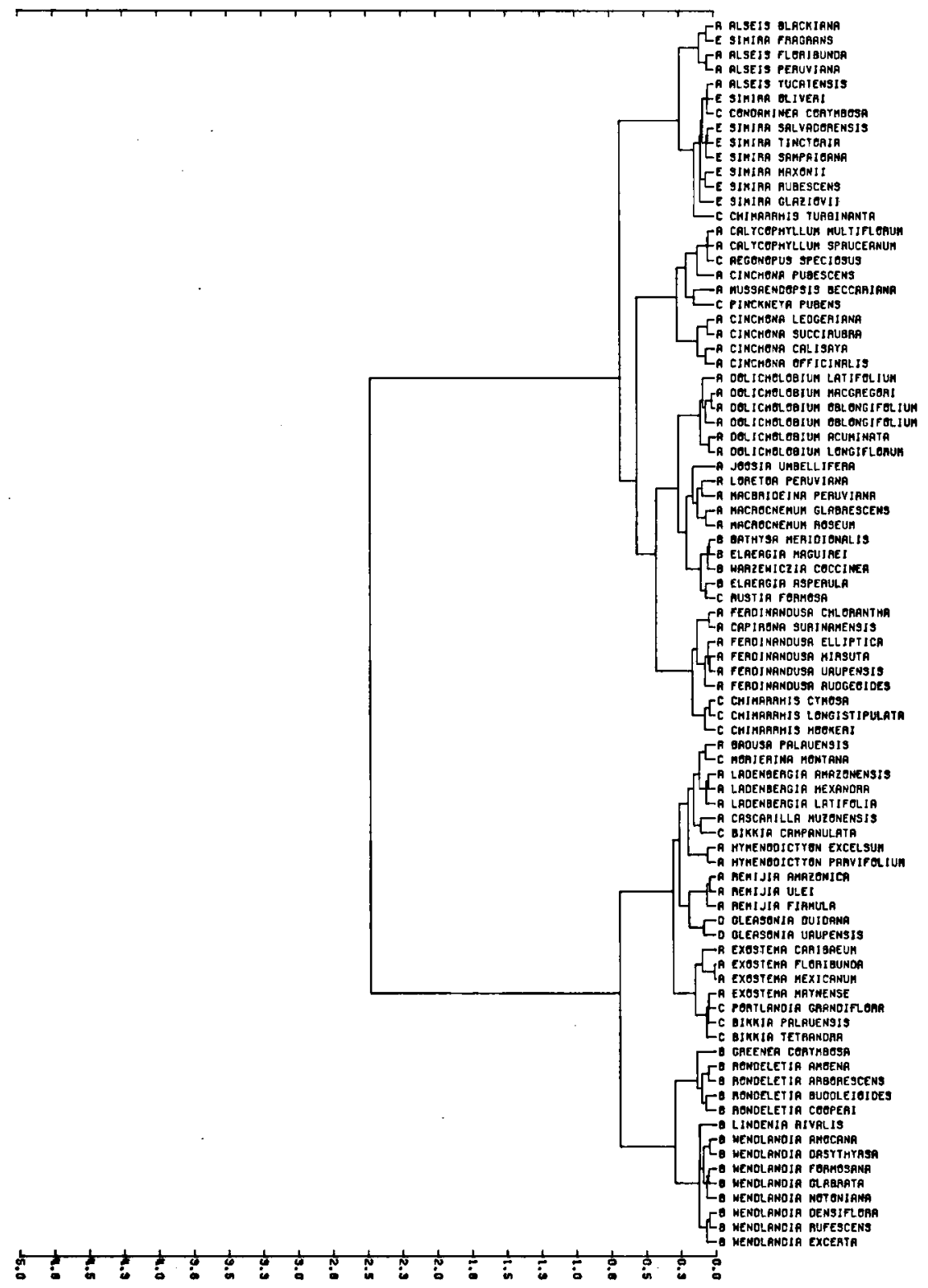

Fig. 2a. Sequence of dendrograms; iterative weighing procedure.
A: Cinchoneae
B: Rondeletieae
C: Condamineae
D: Gleasonia
E: Simira
Rondeletieae sensu lato 


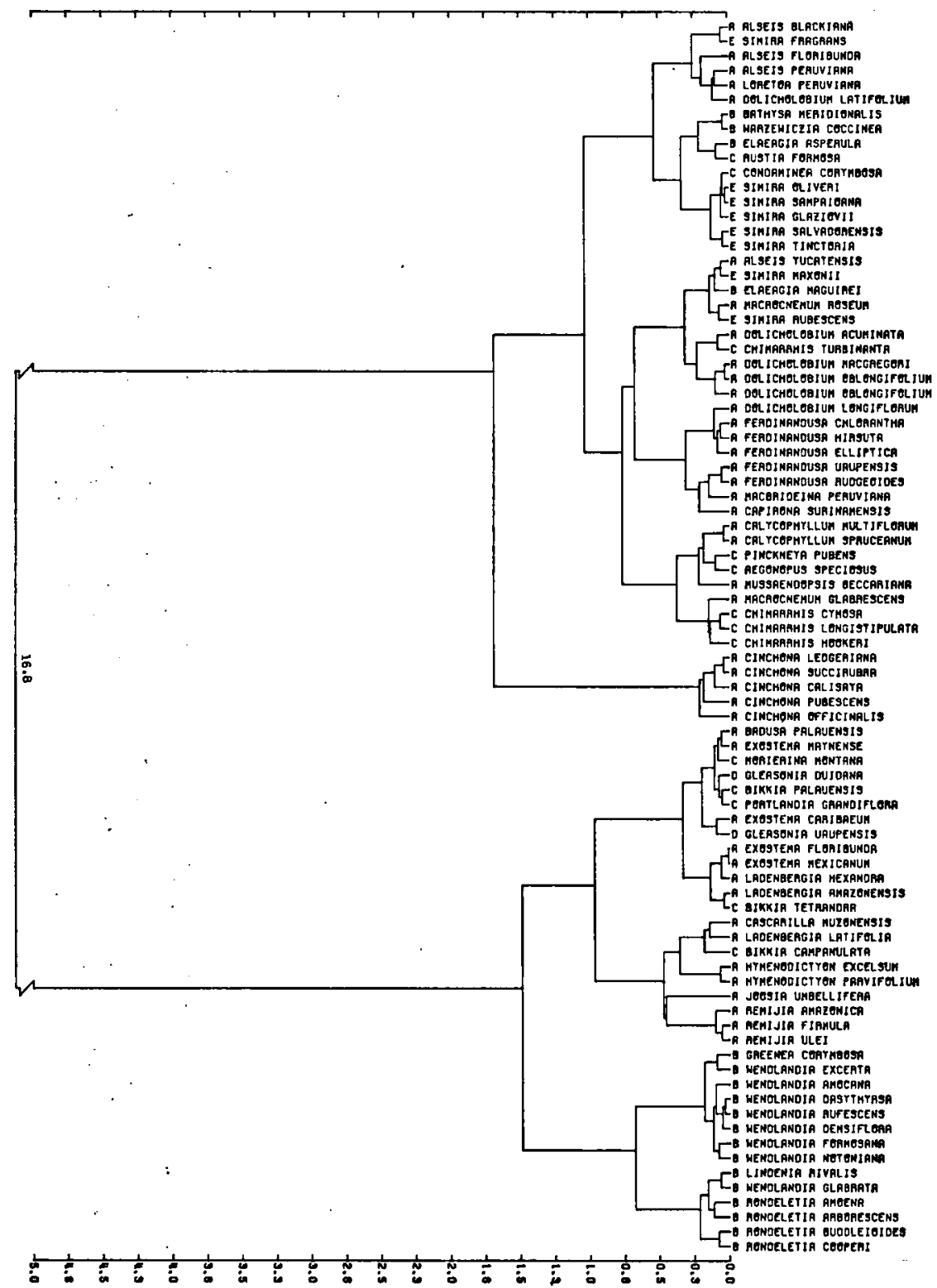

Fig. 2b. Sequence of dendrograms; iterative weighing procedure.
A: Cinchoneae
B: Rondeletieae
C: Condamineae
D: Gleasonia
$>$ Rondeletieae sensu lato
E: Simira 


\title{
OENOROGRAM (WARD AVERAGES) OF MEAN CHARACTER DIFFERENCES IN Q-MODE
}

\author{
RUBIRCEAE. WOODRNATOMY \\ $C Y=2$
}

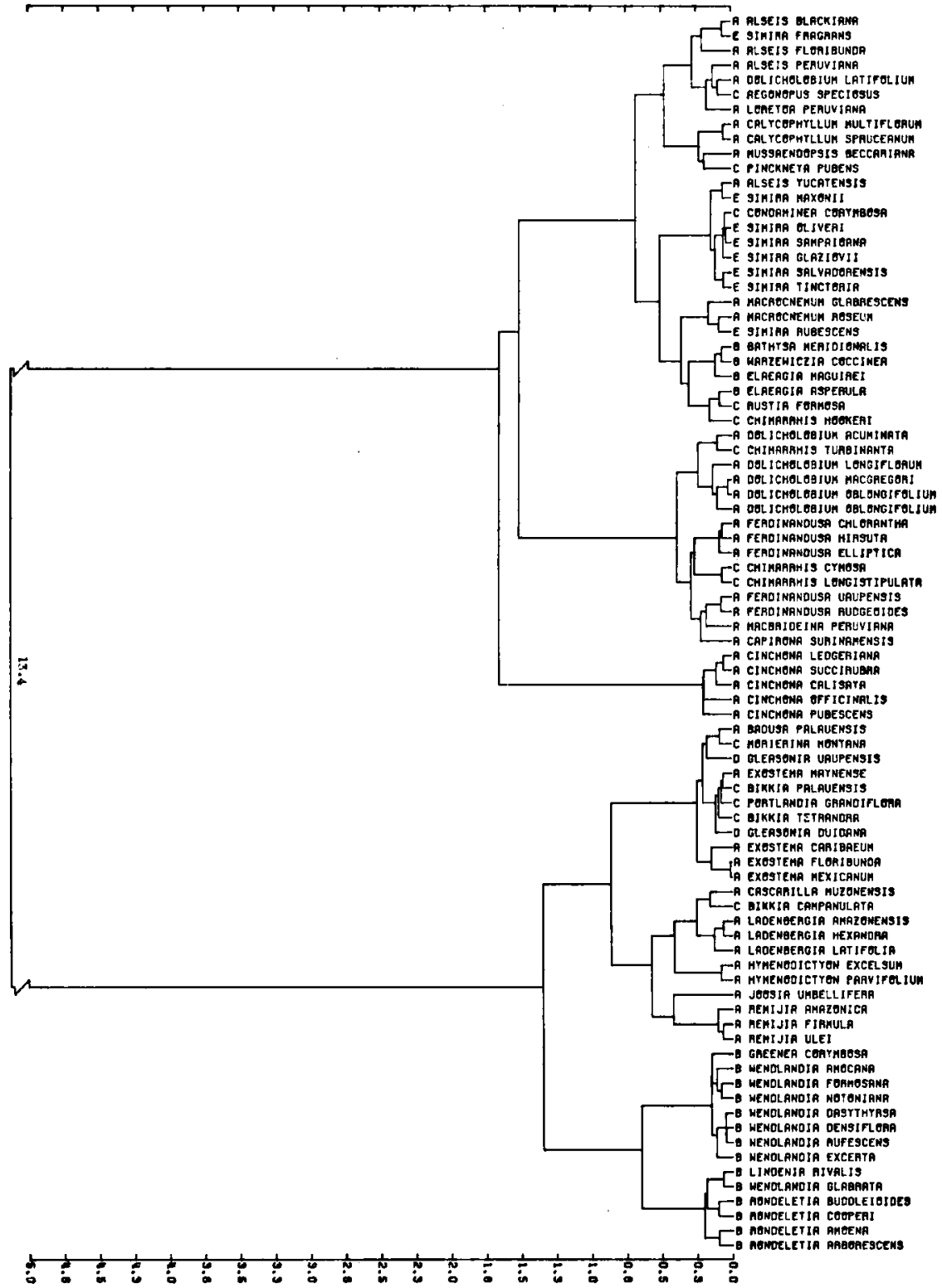

Fig. 2c. Sequence of dendrograms; iterative weighing procedure.
A: Cinchoneae
B: Rondeletieae
C: Condamineae
D: Gleasonia $\}$ Rondeletieae sensu lato
E: Simira 


$$
\text { RUBIACEAE, WOODANATOMY CY=3 }
$$

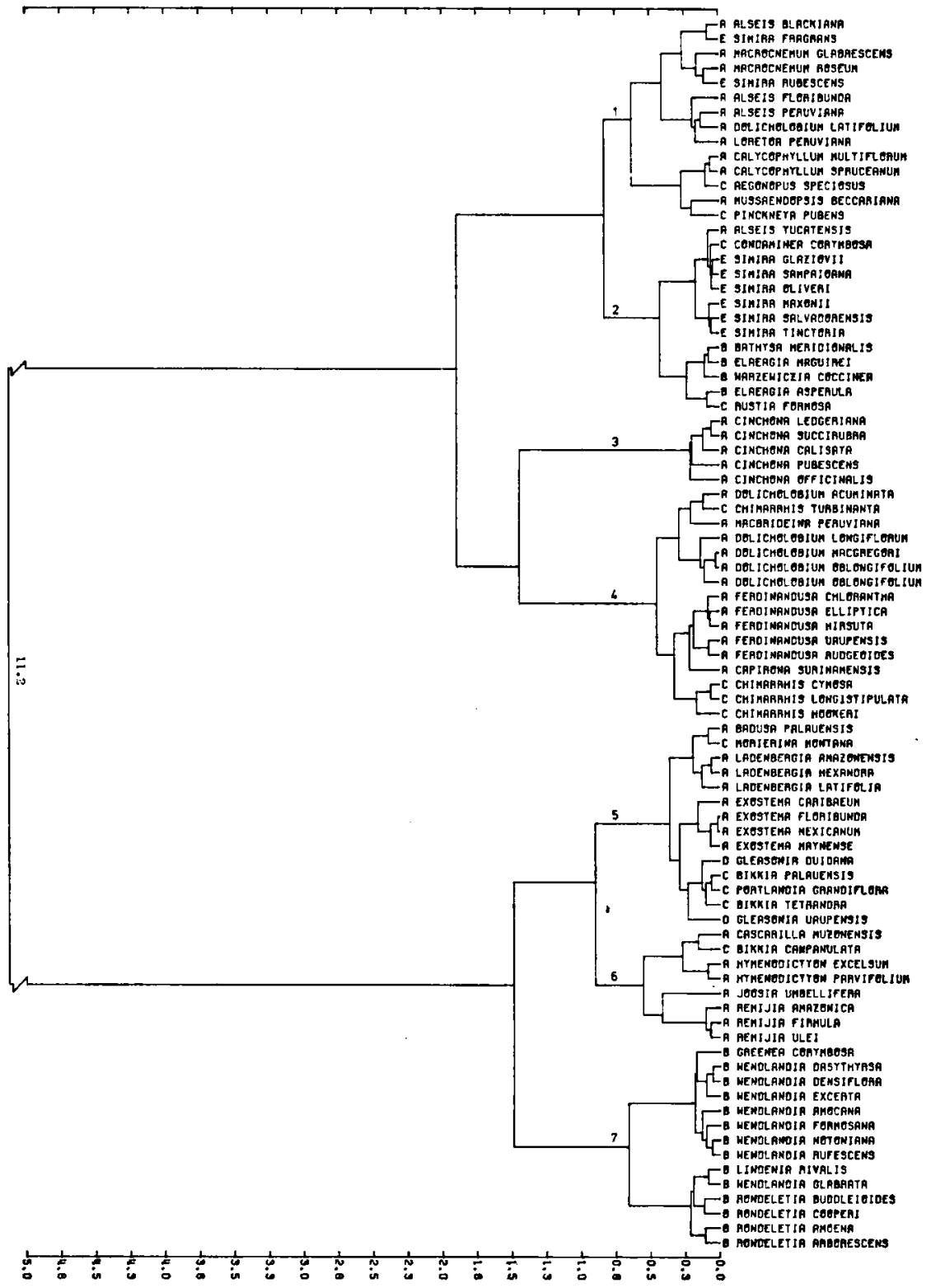

Fig. 2d. Sequence of dendrograms; iterative weighing procedure.
A: Cinchoneae
B: Rondeletieae
C: Condamineae
D: Gleasonia >Rondeletieae sensu lato
E: Simira 


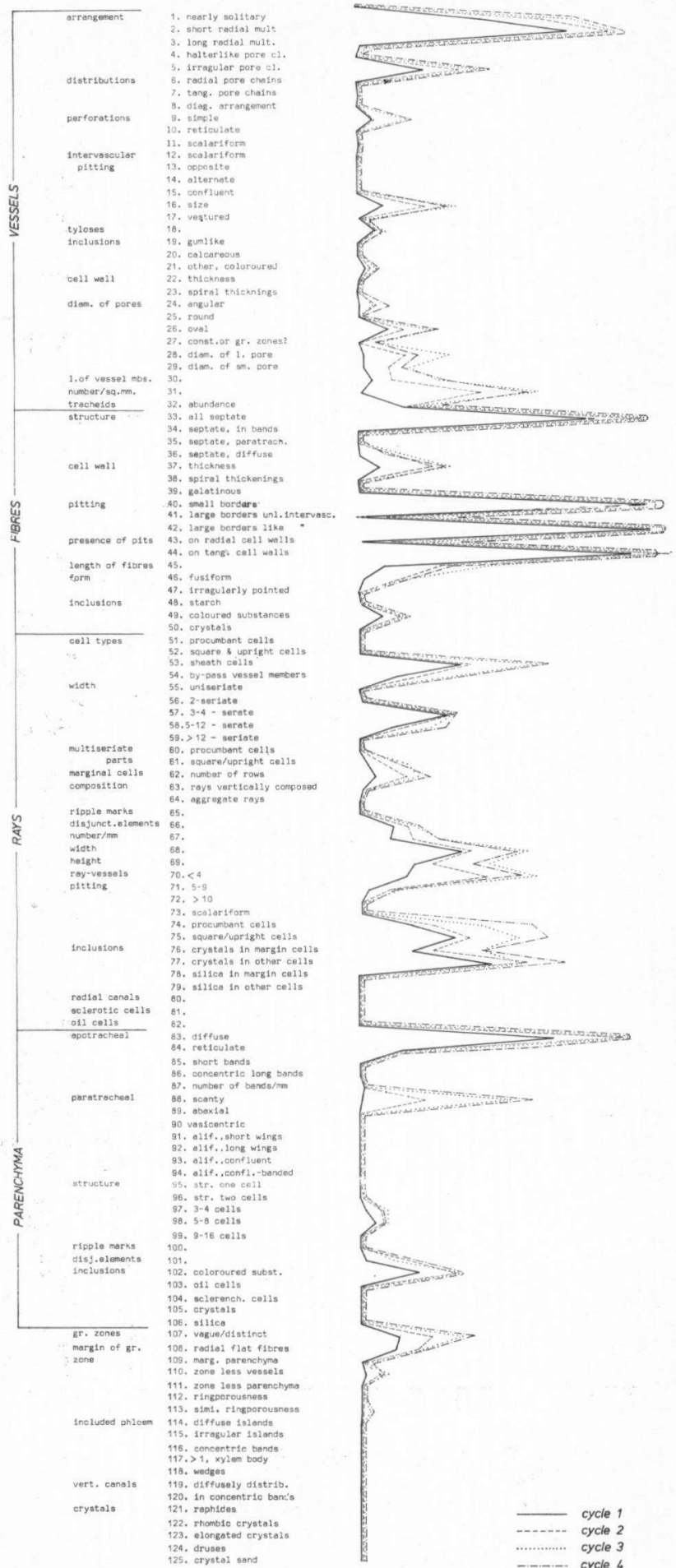

Fig. 3. Profile of character weights belonging to the sequence of dendrograms shown in fig. 2 . 
Step 5

Weights (fig. 3):

Little change in weights occurs.

Dendrogram:

Invariant at the level of 8 clusters except for the transfer of one of the two represented Gleasonia species to the tribe Rondeletieae where they are generally classified. This close affinity, however, has been doubted by BREMEKAMP (1966).

\subsection{Discussion and conclusions drawn from the protocol above}

We want to stress that the results include the sequence of dendrograms, not just the last one. By examining these together with the profile of character weights the characters responsible for changes in pattern may be discovered.

The pattern variation shows only minor changes during the iteration. (This is not generally so as may be seen in HoGEwEG (1975) where is reported that in the Myosotis palustris complex the pattern changes dramatically from an uninterpretable pattern via a pattern consistent with prior classifications to one based on the number of chromosomes.) From the absence of major changes we conclude the absence of a conflicting pattern of comparable strength in other subspaces than the one of the characters which are responsible for the bipartition i.e. the pattern of libriform fibres and fibre tracheids.

The emergence of Cinchona by weighing of the characters connected with the tripartition shows its intermediate character with respect to fibre tracheids and libriform fibres as noted by Koek-Noorman (KoEK-NOORMAN \& Hogeweg 1974).

In order to appreciate this result fully we should keep in mind that the notion libriform fibres and fibre tracheids was not given explicitly to the procedure which received as input the "atomic" observations relevant to this notion (among other characters). Thus the procedure has generated a notion comparable to the one used in the woodanatomical literature (REINDERS 1935) and subdivided the dataset according to this notion.

Another remarkable feature of the iteration is the emergence of the tribe Rondeletieae as a separate cluster in each or the major clusters of the dendrogram (i.e. a group of Rondeletieae with libriform fibres and one with fibre tracheids), as this involves a convergence of the pattern of variation of the woodanatomy to the classifications based on flower morphology. The separation of the Rondeletieae is, however, not perfect (several 'misclassifications' occur) and on a low hierarchical level (the Cinchoneae are subdivided in several groups at higher hierarchical levels than the one separating the Rondeletieae).

It is, however, striking that ambiguities in the existing classifications may be traced in our iteration. Thus we note that Bremekamp has tentatively split off the genera Simira and Gleasonia from the Rondeletieae and placed them in two separate groups. In our iteration Simira joins the other Rondeletieae with libriform fibres only in later steps of the iteration, while only one of the species of the genus Gleasonia joins the Rondeletieae with fibre tracheids finally in the very last step to which the iteration was pursued. 
With regard to the Condamineae no convergence to the existing classifications occurs as they remain scattered all through the dendrogram.

The next question which arises is whether the two subgroups of Rondeletieae have any characteristics in common or just represent incomparable subgroups in our dataset. This question cannot be answered by the method described so far as it only specifies the importance of characters for the separation of groups in the entire dataset and does not generate a characterisation of the clusters other than an extensive definition (i.e. the objects belonging to the cluster).

In the next paragraph we will examine this question by generating new compound features which optimally represent the variation of the clusters which are generated by the iterative weighing procedure.

\section{GENERATION OF FEATURES WHICH OPTIMALLY REPRESENT CLUSTER DISSIMILARITY RELATIONS}

Initially characterisation of the clusters which were generated by the iterative character weighing procedure was attempted in terms of single 'atomic' features, but this did not yield satisfactory results as all clusters were very variable in each of the characters. Only the major bipartition could be characterised in terms of atomic characters (as well as in terms of the wood anatomical notions libriform fibres and fibre tracheids).

We therefore had to try to find compound characters in terms of which the cluster differences could be expressed.

This was done in the following way. The first seven clusters of the final dendrogram ( fig. $2 d$ ) were represented by their centroids. We felt justified to do so because an approximately spherical shape (in the, by weighing transformed, space) was ensured by the clustering method (criterion minimum increase of sum of squares, WARD 1963). Principal component analysis was next performed on the centroids.

The components may be seen as new characters; they are linear combinations of the original characters, independent of each other, and represent the variation in the dataset optimally.

The first component represents, as expected, the differences of the two major clusters. In fig. 4 the horizontal axis shows a separation of groups with fibre tracheids (left) and libriform fibres (right). A large number of other characters support this separation; these are: vessel arrangement (solitary - left, radial multiples and clusters - right), simple vessel end-wall perforations large (left) or small (right), rays 3-4-seriate (left) to rays 3-12-seriate with sheath cells (right), ray-vessel pits $\leqslant 4 \mu \mathrm{m}$ (left) to 5-9 $\mu \mathrm{m}$ (right), presence of crystal sand (right), slightly longer ray margins (left), parenchyma diffuse-reticulate (left), with coloured inclusions (left), and vague growth rings marked by flattened fibres (right). Cinchona occupies, as expected, an intermediate position on this axis (KOEK-NOORMAN \& HOGEWEG 1974).

The second component is very surprising as it splits off both Rondeletieae clusters on the positive side and two subgroups of the Cinchoneae on the nega- 


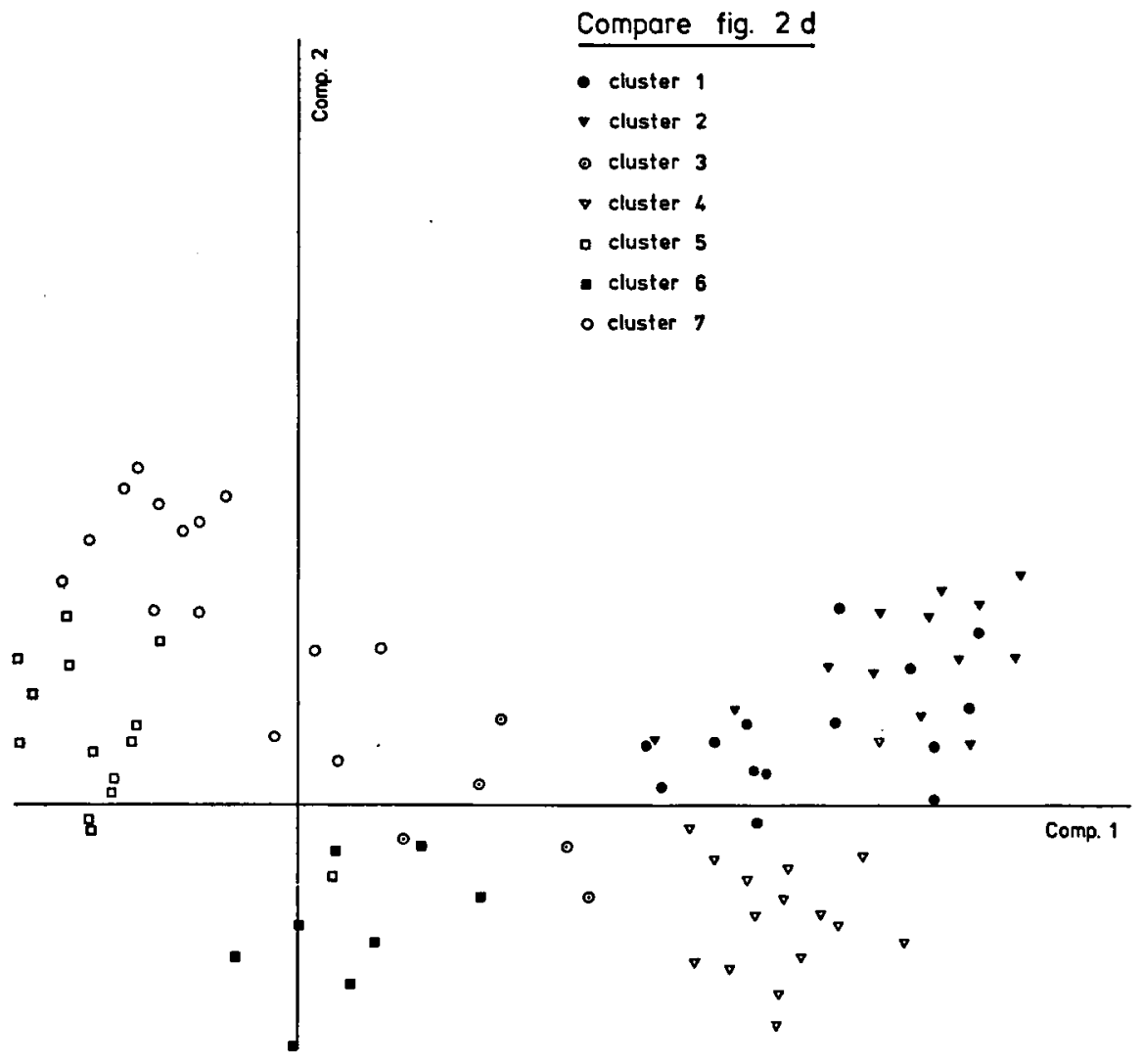

Fig. 4. Scatter diagram on the first two principal components. The horizontal axis represents the first component, the vertical axis the second component. The clusters 2 and 7 consist nearly exclusively of Rondeletieae. Cluster 3 represents the Cinchona species. The clusters $1,4,5$, and 6 consist predominantly of Cinchoneae and Condamineae.

tive side. Thus we see that we have found a pattern of variation of the wood anatomical structure similar to the one found in flower morphology, but that this is not the most conspicuous pattern, and independent of the most conspicuous pattern.

This result was obtained by iterative weighing which caused a superposition of the globally important pattern of the dataset on more local variations as indicated above. It was obtained by an entirely non-supervised method, i.e. independent of the known classifications.

The new feature, as expressed by the character weights on the second component, shows that the Rondeletieae are characterised by numerous small solitary vessels with round diameter, by short vessel elements with large simple end-wall perforations and with coloured inclusions, by intervascular pits $\leqslant 4 \mu \mathrm{m}$, by short fibres with coloured inclusions and by small unilaterally compound rayvessel pits. 
The following points seem moreover of interest and beg for re-examination of the flower morphology of the present groups.

a. The same compound character which splits off the Rondeletieae from the Cinchoneae splits the Cinchoneae into two groups, i.e. the group of Ferdinandusa and Dolicholobium (showing libriform fibres, cluster 4) together with cluster 6 (Cascarilla, Joosia, Remijia and Hymenodictyon) versus the remainder. The first group differs most conspicuously from the Rondeletieae.

b. With respect to the generated feature the genus Simira is more similar to the Rondeletieae with fibre tracheids than the other Rondeletieae with libriform fibres are. This is opposite to the conclusions drawn by Bremekamp who splits off the Simira species.

The third component splits off the genus Cinchona once more, i.e. this genus is not only exceptional in respect to the intermediate nature of the fibres and the other characters correlated therewith but has some other specific characters e.g. low multiseriate rays, diffuse and terminal parenchyma consisting of strands of 2-4 cells.

The fourth component lacks an obvious interpretation. The fifth component should be mentioned because it seems to be responsible for the misclassifications of the Rondeletieae. In this (compound) character several parenchyma characters play a role.

\section{DISCUSSION}

In the present paper we have used the existing classifications of the groups of Rubiaceae under investigation as reference for the comparison of the results. The pattern detection device did, however, not have any knowledge about these classifications, i.e. the pattern of variation of the wood anatomical structure is not molded onto the existing classifications. Independent attempts to do so (by intuitive means or by single character statistics) failed utterly.

It is therefore quite remarkable that a pattern of variation was found which at least partially resembles the pattern of variation as expressed in the existing classifications, as second most important pattern, when our method of iterative character weighing was applied.

The method was designed with, among other things, the example of classical taxonomic practice in mind. The obtained results seem to suggest that we have succeeded in simulating the type of pattern processing as done in classical taxonomy. In our method (and we hypothesize that this is also true for classical taxonomy) "important" characters are generated on the basis of the variation within the dataset, rather than selected on a priori grounds, and the more global pattern of variation in the dataset under consideration is enforced upon the more local variation by weighing the important characters highly.

The enforcement of the more global pattern of variation (evidently strongly dataset dependent) seems to be a fruitful method for describing the pattern of variation of organisms as it yields (at least to a certain extent and in our case as the second most important) a pattern which is consistent in entirely disconnect- 
ed character sets of the same set of objects. The latter being the best (and only) reenforcement systematic studies can ever obtain.

\section{ACKNOWLEDGEMENT}

The authors wish to thank Mr. M. Groeneveld for his accurate assistance in the preparation of the illustrations.

\section{REFERENCES}

BREMEKAMP, C. E. B. (1966): Remarks on the position, the delimitation and the subdivision of the Rubiaceae. Acta Bot. Neerl. 15: 1-33.

Hogeweg, P. (1975): Iterative Character Weighing in Numerical Taxonomy. Pattern Recognition, in the press.

- \& B. HeSPER (1972): BIOPAT, program system for Biological Pattern analysis. Theor. Biol. Group, Univ. of Utrecht.

Kendall, M. G. (1972): Cluster analysis. In S. Watanabe (ed.): Frontiers of Pattern-recognition: 291-310. Acad. Press. New York.

Koek-Noorman, J. (1969): A contribution to the wood anatomy of South American (chiefly Suriname) Rubiaceae. II. Acta Bot. Neerl. 18: 377-395.

- \& P. Hogeweg (1974): The wood anatomy of Vanguerieae, Cinchoneae, Condamineae and Rondeletieae (Rubiaceae). Acta Bot. Neerl. 23: 627-653.

KRUSKAL, W. H. \& W. A. WALlis (1952): Use of ranks in one criterion variance analysis. J. Amer. Statist. Ass. 47: 583-621.

LeENHOUTS, P. W. (1968): A Guide to Herbarium Taxonomy. Regnum Vegetabile 58. IAPT.

REINDERS, E. (1935): Fiber-tracheids, libriform fibres and systematics in woodanatomy. Trop. Woods 44: 30-36.

Schumann, K. (1897): In A. Engler \& K. Prantl, Die natürlichen Pflanzenfamilien. IV (4). Leipzig.

Sokal, R. R. \& P. H. A. Sneath (1963): Principles of Numerical Taxonomy. W. H. Freeman and Comp., Bordeaux.

SNeAth, P. H. A. \& R. R. Sokal (1973): Numerical Taxonomy. W. H. Freeman and Comp., San Francisco.

Steyermark, J. A. (1972): In B. Maguire c.s.: The Botany of the Guyana Highlands. IX. New York Bot. Garden 23: 227-832.

Verdcourt, Рh. D. (1958): Remarks on the Classification of the Rubiaceae. Bull. J. Bot. Brux. 28: 209-290.

WARD, J. H. (1963): Hierarchical Grouping to Optimize an Objective Function. J. Amer. Statist. Ass. 58: 236-244.

WATANABE, S. (1969): Knowing and Guessing, a quantitative study of inference and information. Chpt. 7,8. Wiley, New York. 